\title{
Effectiveness of Ursodeoxycholic Acid in the Treatment of Primary Sclerosing Cholangitis with Ulcerative Colitis: A Pediatric Case
}

\author{
Yuji Fujita, ${ }^{1}$ Takeshi Sugaya, ${ }^{2}$ Ayano Inui ${ }^{3}$ and Shigemi Yoshihara ${ }^{1}$ \\ ${ }^{1}$ Department of Pediatrics, Dokkyo Medical University, Shimotsuga-gun, Tochigi, Japan \\ ${ }^{2}$ Department of Gastroenterology, Dokkyo Medical University, Shimotsuga-gun, Tochigi, Japan \\ ${ }^{3}$ Department of Pediatric Hepatology and Gastroenterology, Saiseikai Yokohamashi Tobu Hospital, Yokohama, \\ Kanagawa, Japan
}

\begin{abstract}
Primary sclerosing cholangitis is a rare disease with poor prognosis that potentially leads to liver cirrhosis and is often complicated by inflammatory bowel disease. Although ursodeoxycholic acid is the most commonly used drug to treat primary sclerosing cholangitis, its effectiveness in treating primary sclerosing cholangitis has not yet been established. An 11-year-old girl had a fever, upper and lower abdominal pain, and bloody stools. Colonoscopy revealed ulcerative colitis. She also had elevated hepatobiliary enzyme levels and C-reactive protein levels, indicating cholangitis after starting food intake, and primary sclerosing cholangitis was diagnosed with endoscopic retrograde cholangiography. Her hepatobiliary enzyme levels gradually improved after ursodeoxycholic acid was started, and symptoms did not recur after food intake. Primary sclerosing cholangitis should be considered if patients, even children, with inflammatory bowel disease, have upper abdominal pain with elevated biliary enzyme levels. The clinical guidelines for primary sclerosing cholangitis treatment have recommended that ursodeoxycholic acid should not be actively used. However, there are some recent reports stating its effectiveness for primary sclerosing cholangitis. In this patient, ursodeoxycholic acid may have been effective for the normalization of the hepatobiliary enzymes. However, it is unknown whether ursodeoxycholic acid improves long-term prognosis. Hence, further evidence regarding the effectiveness of ursodeoxycholic acid in the treatment of primary sclerosing cholangitis needs to be established.
\end{abstract}

Keywords: inflammatory bowel disease; primary sclerosing cholangitis; ulcerative colitis; ursodeoxycholic acid Tohoku J. Exp. Med., 2021 February, 253 (2), 109-112.

\section{Introduction}

Primary sclerosing cholangitis (PSC) is a rare disease with a poor prognosis that might lead to liver cirrhosis. Although certain medical treatments such as ursodeoxycholic acid (UDCA), corticosteroids, and biologics have hitherto been tried, no established treatment has been reported for PSC. Liver transplantation is the only radical treatment; however, the procedure is highly invasive. Despite successful living donor liver transplantation, the disease recurred in many cases (Egawa et al. 2011). Although UDCA is the most commonly used drug to treat PSC, its effectiveness in PSC has not yet been established. Herein, we report a pediatric case of PSC with underlying ulcerative colitis (UC) where high-dose UDCA was useful in treating PSC.

\section{Case Presentation}

An 11-year-old girl had lower abdominal pain, diarrhea, and occasional bloody stool for 3 months. She had a fever and upper abdominal pain for 1 week and was admitted to our hospital. She had elevated alkaline phosphatase (ALP), r-glutamyl transpeptidase (GGT), and C-reactive protein (CRP) levels at the age of 10 years, which had improved with the administration of $\mathrm{H} 2$ receptor blocker. Her father had a history of UC. Physical examination at admission revealed tenderness of the upper and lower abdomen. Her blood tests showed abnormal values: aspartate aminotransferase: $88 \mathrm{IU} / \mathrm{L}$ (reference range 13-30 IU/L),

Received January 7, 2021; revised and accepted January 25, 2021. Published online February 5, 2021; doi: 10.1620/tjem.253.109.

Correspondence: Yuji Fujita, M.D., Department of Pediatrics, Dokkyo Medical University, 880 Kitakobayashi, Mibu, Shimotsuga-gun, Tochigi 321-0293, Japan.

e-mail: fujitay@dokkyomed.ac.jp

(C)2021 Tohoku University Medical Press. This is an open-access article distributed under the terms of the Creative Commons Attribution-NonCommercial-NoDerivatives 4.0 International License (CC-BY-NC-ND 4.0). Anyone may download, reuse, copy, reprint, or distribute the article without modifications or adaptations for non-profit purposes if they cite the original authors and source properly.

https://creativecommons.org/licenses/by-nc-nd/4.0/ 
alanine transaminase: $101 \mathrm{IU} / \mathrm{L}$ (reference range 7-23 IU/ L), alkaline phosphatase: 1,935 IU/L (reference range 3001,380 IU/L), GGT: $512 \mathrm{IU} / \mathrm{L}$ (reference range 9-32 IU/L), total bilirubin: $0.9 \mathrm{mg} / \mathrm{dL}$ (reference range $0.4-1.5 \mathrm{mg} / \mathrm{dL}$ ), CRP: $10.37 \mathrm{mg} / \mathrm{dL}$ (reference range $<0.14 \mathrm{mg} / \mathrm{dL}$ ), immunoglobulin G: $2,881 \mathrm{mg} / \mathrm{dL}$ (reference range $740-1,655 \mathrm{mg} /$ $\mathrm{dL}$ ), immunoglobulin G4: $84.6 \mathrm{mg} / \mathrm{dL}$ (reference range 2.6$104 \mathrm{mg} / \mathrm{dL}$ ), antinuclear antibody titer: 1:40 (reference range $<1: 20$ ), proteinase 3 antineutrophil cytoplasmic antibody: $92.5 \mathrm{IU} / \mathrm{L}$ (reference range $<3.5 \mathrm{IU} / \mathrm{mL}$ ), and antismooth muscle antibody titer: 1:40 (reference range $<1: 40$ ). Bacterial culture of blood and feces showed no pathogenic bacteria.

Her symptoms and laboratory findings improved with fasting after admission. Colonoscopy showed edema and absent vascular pattern through the entire colon (Fig. 1). We diagnosed UC with total colonic involvement based on colonoscopic (Fig. 1) and pathological findings. Esophagogastroduodenoscopy and small intestine capsule endoscopy showed no abnormal findings. Mesalazine (60 $\mathrm{mg} / \mathrm{kg} /$ day) was administered as a primary treatment and food intake also started from Day 15. However, she had a fever, upper abdominal pain, and elevated hepatobiliary enzyme levels, and her CRP levels worsened on Day 20. The lower abdominal pain and bloody stool were not seen (Fig. 2). Abdominal ultrasonography showed biliary sludge in the common bile duct. Cholangitis was suspected, and ceftriaxone was administered for 5 days from Day 20. On Day 24 we then performed endoscopic retrograde cholangiography (ERC), which revealed strictures of the common and intrahepatic bile ducts, indicating PSC (Fig. 3). Bile outflow from the duodenal papilla was good; hence, neither balloon expansion nor balloon stenting was performed. The bacterial culture of bile was negative. No tumor cells were found in bile cytodiagnosis. Liver biopsy revealed PSC that was not complicated with autoimmune hepatitis.

She had cholangitis again from Day 30 after food intake was resumed on Day 28. Therefore, food intake was stopped, and a high-dose UDCA (30 mg/kg/day) regimen with ceftriaxone was started from Day 32. Following this treatment, food intake did not trigger cholangitis. Abdominal ultrasonography revealed the disappearance of biliary sludge in the common bile duct on Day 45 . However, she had lower abdominal pain and bloody stools from Day 46 with increased food intake. Colonoscopy revealed absent vascular pattern and marked erythema through the entire colon, indicating exacerbation of UC, for which prednisolone $(1 \mathrm{mg} / \mathrm{kg} /$ day $)$ was administered from Day 51. Following this, her symptoms rapidly improved, and no symptoms resurfaced after resuming a normal diet. She had normal GGT levels 2 months after starting UDCA and has normal hepatobiliary enzyme levels for 1 year. Informed consent for publishing this case report was obtained from the patient's parents.

\section{Discussion}

PSC is a rare hepatobiliary disease in adult patients, and rarer in children (less than $20 \%$ of that in adults) (Card et al. 2008; Yokoda and Carey 2019); it is occasionally complicated with IBD, and especially by UC. Although some patients may be asymptomatic for PSC, symptoms such as fever and upper abdominal pain indicating cholangitis may also be caused by stenosis of the biliary duct, as in the present case (Hirschfield et al. 2013). At the time of admission, we did not consider the possibility that cholangitis might be causing upper abdominal pain that was recurrently triggered by food intake. PSC should be considered in patients, even in pediatric patients, if they have IBD with

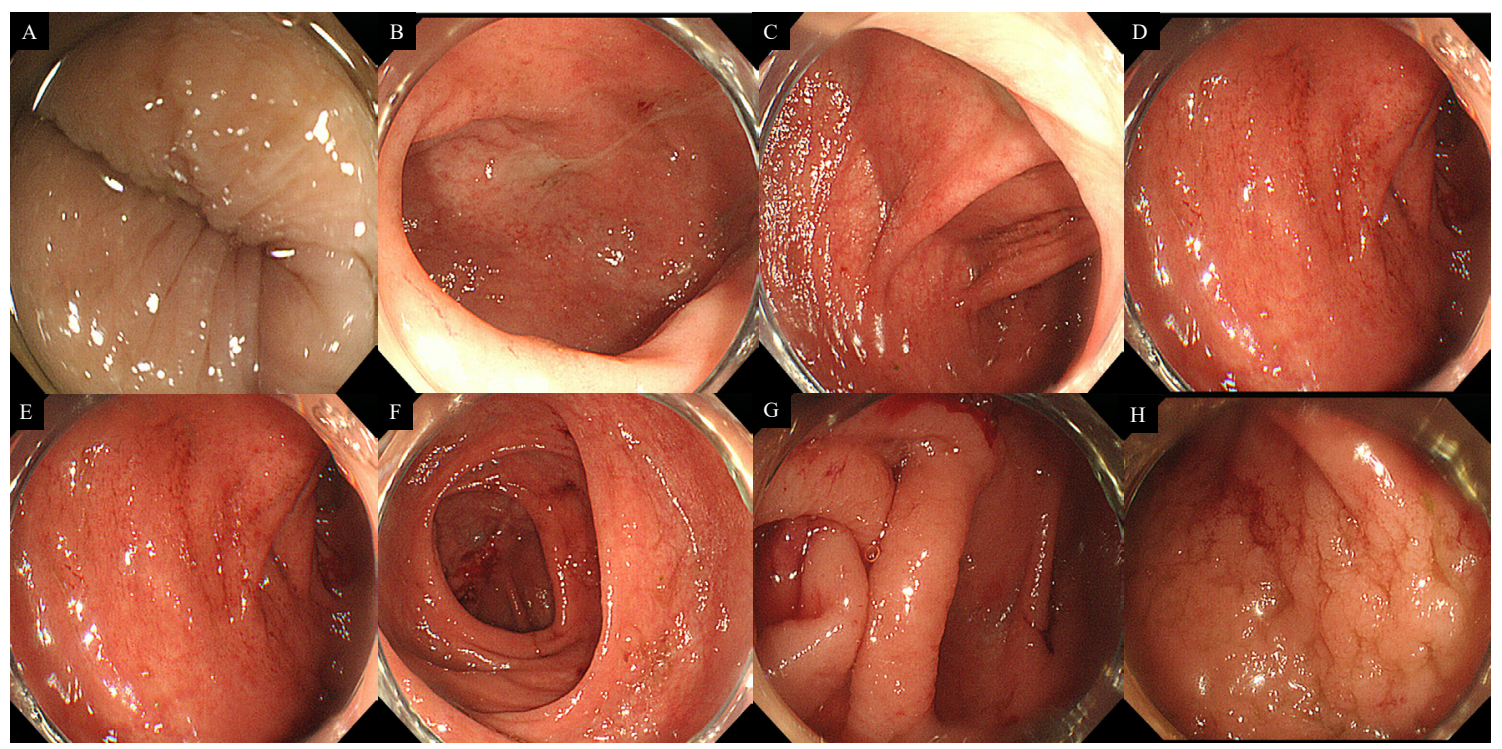

Fig. 1. Colonscopic image at the time of the admission. Colonoscopy showed edema and absent vascular pattern through the entire colon. (A) Anal, (B) rectum, (C) sigmoidal colon, (D) descending colon, (E) transverse colon, (G) ascending colon, $(\mathrm{G})$ cecum, and $(\mathrm{H})$ ileocecum. 


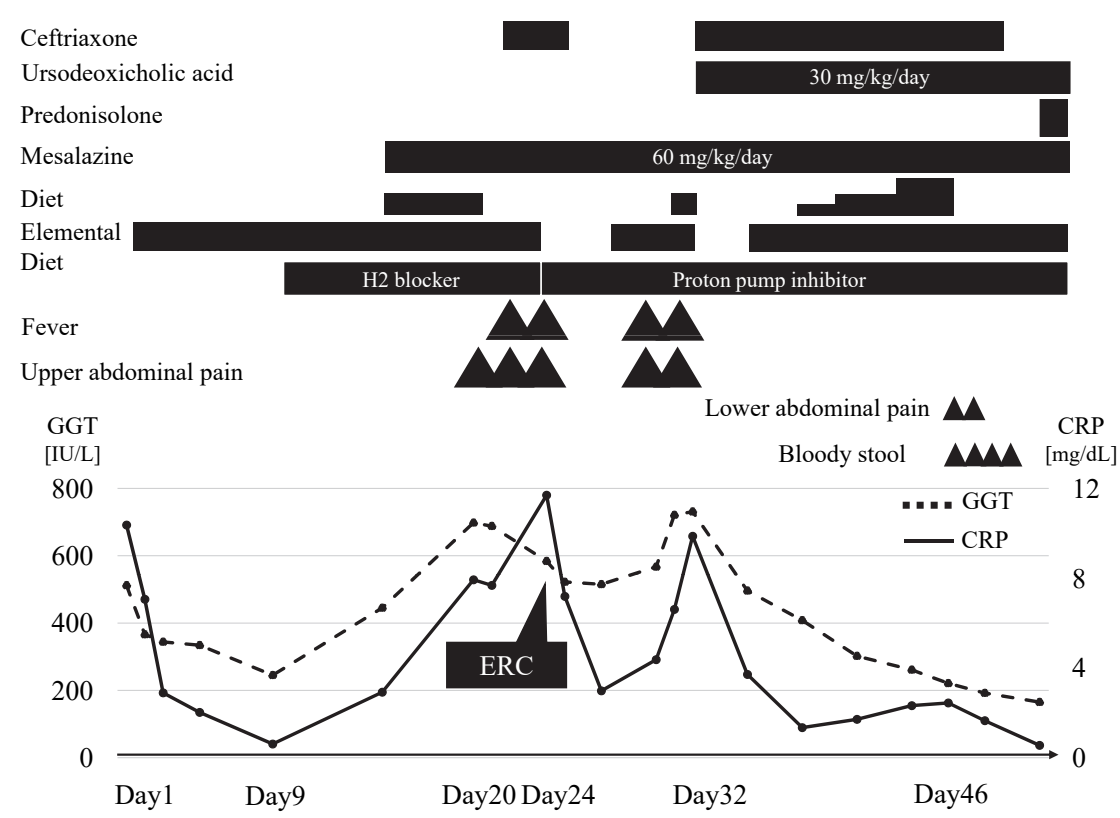

Fig. 2. Patient's course of treatment after hospital admission. She was diagnosed ulcerative colitis (UC) with colonoscopic and pathological findings on Day 8. She had cholangitis from Day 20. Primary sclerosing cholangitis was diagnosed base on endoscopic retrochorangiographic finding on Day 24. She had cholangitis again from Day 30 after food intake was resumed on Day 28. High-dose ursodeoxycholic acid was started from Day 32. Following ursodeoxycholic acid treatment, food intake did not trigger cholangitis. She had exacerbation of UC from Day 46, for which prednisolone was administered from Day 51.

CRP, C-reactive protein; ERC, endoscopic retrograde cholangiography; GGT, $\gamma$-glutamyl transpeptidase.

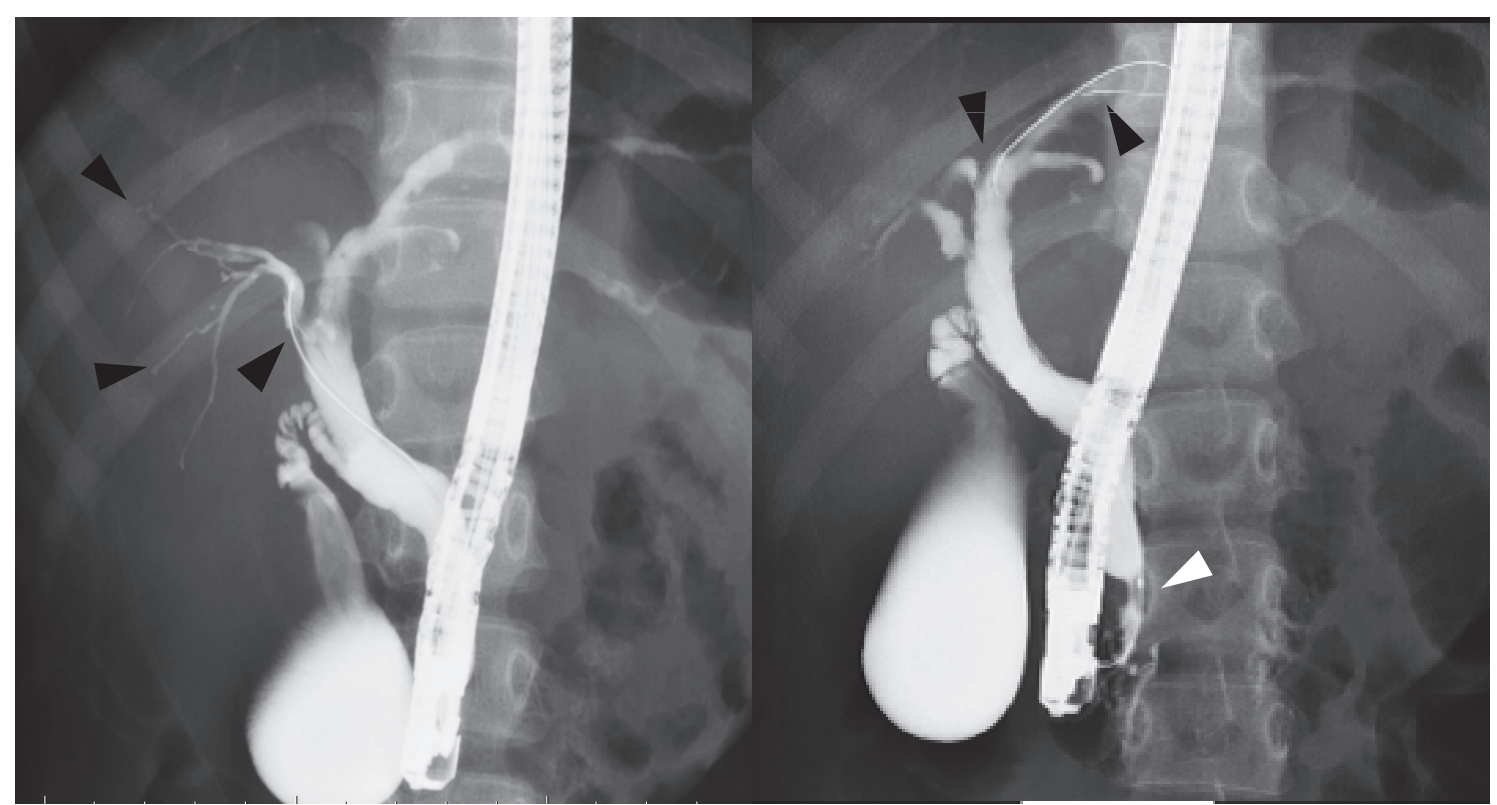

Fig. 3. Endoscopic retrochorangiographic image. Endoscopic retrochorangiography revealed strictures of the common (a white arrow) and intrahepatic bile ducts (black arrows).

ERC, endoscopic retrochorangiography.

upper abdominal pain and elevated biliary enzyme levels.

Recurrent cholangitis leads to disease progression of PSC. Therefore, early diagnosis of PSC and rapid clinical intervention for cholangitis are essential to achieve favorable outcomes (Pohl et al. 2006). The frequent use of anti- biotics in recurrent cholangitis may lead to the risk of multiple antibiotics resistance. In this case, the patient's common bile duct had stenosis, and balloon dilatation with or without stent placement is also often needed in some cases. However, bile outflow from the duodenal papilla 
was good; therefore, balloon dilatation was not required. It might be possible that the antibiotic course was effective. However, antibiotics were prescribed during the first episode and did not have any notable impact. Her cholangitis did not recur after UDCA, coupled with antibiotics was administered during the second cholangitis. This result may indicate that UDCA can be useful for treating cholangitis.

Although UDCA is the most commonly used drug to treat PSC, its effectiveness has not yet been established. Moreover, the clinical guidelines for PSC treatment have recommended that UDCA should not be actively used (Lindor et al. 2015). While UDCA does not effectively improve prognosis, reports suggest that it improves the levels of the laboratory markers ALP and GGT (Lindor et al. 2009). Deneau et al. reported that UDCA might improve prognosis in patients with normalized biliary enzyme levels, and it should be stopped in cases where no improvement of biliary enzyme levels (Deneau et al. 2019). In a retrospective study of pediatric PSC, high-dose UDCA was used only in the group with a good prognosis (Umetsu et al. 2019). It was also reported that complication of autoimmune hepatitis in PSC is a poor prognostic factor. Autoimmune hepatitis was absent in our case.

We believe that UDCA might have played a role in normalizing the hepatobiliary enzyme levels in our patient. However, it is unknown whether it improves long-term prognosis. Further evidence regarding the effectiveness of UDCA in the treatment of PSC needs to be established.

\section{Acknowledgments}

We would like to thank Editage (https://www.editage. com) for English language editing.

\section{Author Contributions}

Dr. Fujita drafted and revised the initial manuscript. Dr. Sugaya, Dr. Inui, and Prof. Yoshihara critically revised the manuscript for important intellectual content. All authors read and approved the final manuscript.

\section{Conflict of Interest}

The authors declare no conflict of interest.

\section{References}

Card, T.R., Solaymani-Dodaran, M. \& West, J. (2008) Incidence and mortality of primary sclerosing cholangitis in the UK: a population-based cohort study. J. Hepatol., 48, 939-944.

Deneau, M., Perito, E., Ricciuto, A., Gupta, N., Kamath, B.M., Palle, S., Vitola, B., Smolka, V., Ferrari, F., Amir, A.Z., Miloh, T., Papadopoulou, A., Mohan, P., Mack, C., Kolho, K.L., et al. (2019) Ursodeoxycholic acid therapy in pediatric primary sclerosing cholangitis: predictors of gamma glutamyltransferase normalization and favorable clinical course. J. Pediatr., 209, 92-96. e1.

Egawa, H., Ueda, Y., Ichida, T., Teramukai, S., Nakanuma, Y., Onishi, S. \& Tsubouchi, H. (2011) Risk factors for recurrence of primary sclerosing cholangitis after living donor liver transplantation in Japanese registry. Am. J. Transplant., 11, 518-527.

Hirschfield, G.M., Karlsen, T.H., Lindor, K.D. \& Adams, D.H. (2013) Primary sclerosing cholangitis. Lancet, 382, 15871599.

Lindor, K.D., Kowdley, K.V. \& Harrison, M.E.; American College of Gastroenterology (2015) ACG clinical guideline: primary sclerosing cholangitis. Am. J. Gastroenterol., 110, 646-659; quiz 660 .

Lindor, K.D., Kowdley, K.V., Luketic, V.A., Harrison, M.E., McCashland, T., Befeler, A.S., Harnois, D., Jorgensen, R., Petz, J., Keach, J., Mooney, J., Sargeant, C., Braaten, J., Bernard, T., King, D., et al. (2009) High-dose ursodeoxycholic acid for the treatment of primary sclerosing cholangitis. Hepatology, 50, 808-814.

Pohl, J., Ring, A., Stremmel, W. \& Stiehl, A. (2006) The role of dominant stenoses in bacterial infections of bile ducts in primary sclerosing cholangitis. Eur. J. Gastroenterol. Hepatol., 18, 69-74.

Umetsu, S., Notohara, K., Nakazawa, T., Tsunoda, T., Sogo, T., Komatsu, H., Tanaka, A., Tazuma, S., Takikawa, H., Inui, A. \& Fujisawa, T. (2019) Long-term outcomes of pediatric-onset primary sclerosing cholangitis: a single-center experience in Japan. Hepatol. Res., 49, 1386-1397.

Yokoda, R.T. \& Carey, E.J. (2019) Primary biliary cholangitis and primary sclerosing cholangitis. Am. J. Gastroenterol., 114, 1593-1605. 